\title{
5D super Yang-Mills theory and the correspondence to $\mathrm{AdS}_{7} / \mathrm{CFT}_{6}$
}

\author{
Joseph A. Minahan, Anton Nedelin* and Maxim Zabzine \\ Department of Physics and Astronomy, Uppsala university, \\ Box 516, SE-75120 Uppsala, Sweden \\ E-mail: joseph.minahan@physics.uu.se, anton.nedelin@physics.uu.se, \\ maxim.zabzine@physics.uu.se
}

In this contribution we present our recent results on $5 D$ super Yang-Mills theory and its relation with $6 D(2,0)$ superconformal theory. We discuss how the $N^{3}$ behavior of the $5 D$ super YangMills free energy can be derived with the use of the localization technique. We show that results obtained from localization in $5 D$ super Yang-Mills are consistent with the corresponding results for compactified $6 D$ theory when we fix the mass of the hypermultiplet in $5 D$ and modify the flat-space relation between the Yang-Mills coupling constant and the radius of compactification.

The European Physical Society Conference on High Energy Physics Stockholm,Sweden

18-24 July, 2013

\footnotetext{
* Speaker.
} 


\section{Introduction}

Among superconformal field theories, the most interesting and strange are the 6-dimensional $(2,0)$ theories. They preserve 16 supersymmetries with $S O(5) R$-symmetry in flat space, thus being maximally supersymmetric. These theories arise as a world-volume theory of M5-branes. But what makes them even more attractive for study are the wide variety of compactifications of these theories. For example, compactification on the torus $T^{2}$ reduces it to $\mathscr{N}=4$ super Yang-Mills, and compactification on the circle $S^{1}$ leads to $5 D$ maximally supersymmetric Yang-Mills (MSYM) $[1,2]$.

The field content of $6 D(2,0)$ theories includes five scalars, four chiral fermions and a 2form potential $B$ with self-dual strength $H=d B=\star d B$. The presence of this last field makes a Lagrangian description of these theories problematic, if not impossible. Moreover, being isolated fixed points of RG flows in 6 dimensions, they have neither a dimensionful nor a dimensionless parameter. All this makes $6 \mathrm{D}(2,0)$ theories very difficult to describe and to study.

One possibility left is to use the conjecture of duality between $6 D(2,0)$ theories and $M$-theory on an $A d S_{7} \times S^{4}$ background. In the large $N$ limit $M$-theory reduces to supergravity, which we can use to calculate different observables, even without having a Lagrangian description for the theory we study. One of the most important results obtained in this way is $N^{3}$ behavior of the free energy and conformal anomaly [3, 4].

Another possible way to study these theories is through their compactifications. As we have mentioned above, $(2,0)$ theory on the circle reduces to $5 D$ MSYM. The relation between the circle radius $R_{6}$ and the coupling constant $g_{Y M}$ follows from the mapping of Kaluza-Klein modes of $(2,0)$ theories to the instanton particles of $5 D$ MSYM [2]:

$$
R_{6}=\frac{g_{Y M}^{2}}{8 \pi^{2}}
$$

Recently it has been proposed that $5 D$ MSYM contains all degrees of freedom of the $(2,0)$ theories $[5,6,7]$. This proposal allow us to use $5 D$ MSYM to define the $(2,0)$ theories and study them. This idea seems strange, since $5 D$ SYM is a non-renormalizable theory, so it needs extra degrees of freedom for a UV completion. To overcome this problem in [5] it was proposed that $5 D$ SYM is finite and thus does not need extra degrees of freedom. In this case it should reveal the same $N^{3}$ behavior of free energy as $(2,0)$ theories do. Recently it has been shown that $5 D$ SYM is divergent at six loops [8], so this cannot be the whole story.

However, in this contribution we will consider recent results $[9,10]$ on $5 D$ MSYM partition function. Using results of the supersymmetric localization we will show that partition function of $5 D$ MSYM indeed has $N^{3}$ behavior. The ingredient essential for this behavior is the presence of a hypermultiplet in the adjoint representation. Moreover, we will show that with a proper choice of the hypermultiplet mass and a renormalisation of the coupling constant, we can reproduce not only a qualitative but even a quantitative picture of the $5 D / 6 D$ correspondence.

\section{Free energy of $5 D \mathrm{SYM}$}

In this section we will briefly derive the free energy of $5 D \mathrm{SYM}$ on the sphere $S^{5}$. In flat 
space we can have $\mathscr{N}=1 \mathrm{SYM}$ preserving 8 supercharges enhanced to maximally supersymmetric $\mathscr{N}=2 \mathrm{SYM}$. The latter case has the $\mathscr{N}=1$ vector multiplet as well as a massless $\mathscr{N}=1$ adjoint hypermultiplet. Putting 5D SYM on the sphere cannot be done in a canonical way as it is not a conformal theory. However SYM with arbitrary hypermultiplet on $S^{5}$ has been constructed recently by adding proper terms to the Lagrangian [11]. As was shown in [12], in order for this theory to preserve 16 supercharges we should consider the adjoint hypermultiplet with mass $M=1 / 2 r$, where $r$ is the radius of $S^{5}$. We will call theories with massive adjoint hypermultiplet $\mathscr{N}=1^{*}$ SYM.

In order to compute the free energy we will use supersymmetric localization [13]. This is a very powerful technique, that allows one to reduce path integrals of supersymmetric observables to matrix integrals, which in many cases are much easier to analyse. Unfortunately, this technique is applicable only for supersymmetric observables, which leaves us only two possible observables in $5 D$ SYM theory: its free energy and a supersymmetric Wilson loop, which we consider in detail in the next section.

The partition function for $5 D \mathscr{N}=1 \mathrm{SYM}$ with gauge group $G$ and massive hypermultiplet in an arbitrary representation $R$ is given by the following expression [11, 14, 15, 12]:

$$
Z=\int_{\text {Cartan }}[d \phi] e^{-\frac{8 \pi^{3} r}{g_{Y}^{2}} \operatorname{Tr}\left(\phi^{2}\right)-\frac{\pi k}{3} \operatorname{Tr}\left(\phi^{3}\right)} Z_{1-\text { loop }}^{\text {vect }}(\phi) Z_{1-\text { loop }}^{\text {hyper }}(\phi)+\mathscr{O}\left(e^{-\frac{16 \pi^{3} r}{g_{Y M}^{2}}}\right),
$$

where 1-loop contributions are given by

$$
\begin{aligned}
& Z_{1-\text { loop }}^{\text {vect }}(\phi)=\operatorname{det}_{\text {Adj }}\left(\sin (i \pi \phi) e^{\frac{1}{2} f(i \phi)}\right) \\
& Z_{1-\text { loop }}^{\text {hyper }}(\phi)=\operatorname{det}_{R}\left(e^{-\frac{1}{4} f\left(\frac{1}{2}-i m-i \phi\right)-\frac{1}{4} l\left(\frac{1}{2}-i m-i \phi\right)+(\phi \rightarrow-\phi)}\right)
\end{aligned}
$$

Here $m$ stands for the dimensionless mass of the hypermultiplet $m=i r M$ and the detailed properties of the special functions $l(z)$ and $f(z)$ can be found in [10].

As we wish to relate our result to supergravity calculation of the $6 D$ theory we take the gauge group to be $S U(N)$ with the hypermultiplet in the adjoint representation and then take large $N$ limit of (2.1). In this limit the partition function is governed by the saddle-point equation

$$
\begin{aligned}
\frac{16 \pi^{3} N}{\lambda} \phi_{i}=\pi \sum_{j \neq i}[(2 & \left.-\left(\phi_{i}-\phi_{j}\right)^{2}\right) \operatorname{coth}\left(\pi\left(\phi_{i}-\phi_{j}\right)\right) \\
& +\frac{1}{2}\left(\frac{1}{4}+\left(\phi_{i}-\phi_{j}-m\right)^{2}\right) \tanh \left(\pi\left(\phi_{i}-\phi_{j}-m\right)\right) \\
& \left.+\frac{1}{2}\left(\frac{1}{4}+\left(\phi_{i}-\phi_{j}+m\right)^{2}\right) \tanh \left(\pi\left(\phi_{i}-\phi_{j}+m\right)\right)\right],
\end{aligned}
$$

where $\phi_{i}$ are the eigenvalues of the $\phi$ matrix. In the expression above $\lambda=g_{Y M}^{2} N / r$ is the 't Hooft coupling constant.

In the weak coupling limit $\lambda \ll 1$ this equation reduces to

$$
\frac{16 \pi^{3} N}{\lambda} \phi_{i} \approx 2 \sum_{j \neq i} \frac{1}{\phi_{i}-\phi_{j}}
$$


whose solution for the density of eigenstates is the well-known Wigner semicircle distribution:

$$
\rho(\phi) \equiv \frac{1}{N} \frac{d n}{d \phi}=\frac{2}{\pi \phi_{0}^{2}} \sqrt{\phi_{0}^{2}-\phi^{2}} ; \quad \phi_{0}=\sqrt{\frac{\lambda}{4 \pi^{3}}},
$$

from which we obtain the following behavior of the free energy:

$$
F=-\log Z \approx-N^{2} \log \sqrt{\lambda} .
$$

In the strong coupling limit $\lambda \gg 1$ we can assume that a generic separation between the eigenvalues is of order $\lambda$ so that $\left|\phi_{i}-\phi_{j}\right| \gg 1$. Under this assumption our saddle point equations (2.4) turn into

$$
\frac{16 \pi^{3} N}{\lambda} \phi_{i}=\pi\left(\frac{9}{4}+m^{2}\right) \sum_{j \neq i} \operatorname{sign}\left(\phi_{i}-\phi_{j}\right) .
$$

Assuming that the eigenvalues $\phi_{i}$ are ordered, we get the solution

$$
\phi_{i}=\frac{\left(9+4 m^{2}\right) \lambda}{64 \pi^{2} N}(2 i-N),
$$

which corresponds to an eigenvalue density

$$
\begin{array}{rlrl}
\rho(\phi) & =\frac{32 \pi^{2}}{\left(9+4 m^{2}\right) \lambda} & |\phi| \leq \phi_{m}, & \phi_{m}=\frac{\left(9+4 m^{2}\right) \lambda}{64 \pi^{2}} \\
& =\begin{array}{cll}
0 & & |\phi|>\phi_{m} .
\end{array}
\end{array}
$$

Substituting this solution back into the partition function leads to the following result for free energy in strong coupling:

$$
F \equiv-\log Z \approx-\frac{g_{Y M}^{2} N^{3}}{96 \pi r}\left(\frac{9}{4}+m^{2}\right)^{2} .
$$

As we can see, $5 D$ SYM indeed has $N^{3}$ behavior for the free energy at large $N$ and strong coupling. This analytical result is consistent with numerical results [10], which supports the approximation of large separations we have used in our calculations. In [10] we have also shown numerically that $\mathscr{N}=1 \mathrm{SYM}$ with only a vector multiplet does not have $N^{3}$ behavior for the free energy, thus leading us to conclude that the presence of an adjoint hypermultiplet is essential for this kind of free energy behavior.

\section{Supersymmetric Wilson loop}

Now to make our result even stronger and move from qualitative to quantitative conclusions we will compare results for the matrix model and supergravity for the other observable. Namely, the supersymmetric Wilson loop, where we can also use localization. To preserve supersymmetry the Wilson loop should wrap the equator of $S^{5}$. In analogy with [13] we write down the expectation value of Wilson loop in the following form: 


$$
\langle W\rangle=\frac{1}{N}\left\langle\operatorname{Tr} e^{2 \pi \phi_{i}}\right\rangle .
$$

Then at strong coupling by using the approximation of large separation between the eigenvalues we get

$$
\langle W\rangle \sim \frac{1}{N} \int \prod_{i} d \phi_{i} \sum_{i} e^{2 \pi \phi_{i}} e^{-\frac{8 \pi^{3} N}{\lambda} \sum_{i} \phi_{i}^{2}+\frac{\pi}{2}\left(\frac{9}{4}+m^{2}\right) \sum_{j \neq i} \sum\left|\phi_{i}-\phi_{j}\right|} .
$$

From this expression it is clear that each of the exponents in the sum will give a negligible contribution to the position of saddle point. Thus we can rewrite the Wilson loop expectation value in the following form:

$$
\langle W\rangle=\int d \phi \rho(\phi) e^{2 \pi \phi},
$$

where $\rho(\phi)$ is the eigenvalue density given by expressions (2.10) and (2.6) for respectively strong and weak coupling. Using these expressions for the density we get:

$$
\langle W\rangle \approx \exp \left(\frac{\lambda}{8 \pi}\right),
$$

at weak coupling, while at strong coupling, using the eigenvalue distribution in (2.10), we find

$$
\langle W\rangle \sim \exp \left(\frac{\lambda}{8 \pi}\left(\frac{9}{4}+m^{2}\right)\right) .
$$

\section{Comparison with supergravity calculations}

In this section we compare our strong coupling results (2.11) and (3.5) with the corresponding results in $(2,0)$ theory obtained with supergravity. We will consider supergravity on $A d S_{7} \times S^{4}$ with AdS boundary chosen to be $S^{1} \times S^{5}$, in order to obtain SYM on $S^{5}$ after an $S^{1}$ compactification. The radii of $A d S_{7}$ and $S^{4}$ are $\ell$ and $\ell / 2$ respectively, where $\ell=2 \ell_{p l}(\pi N)^{1 / 3}$. The $A d S_{7}$ metric is given by:

$$
d s^{2}=\ell^{2}\left(\cosh ^{2} \rho d \tau^{2}+d \rho^{2}+\sinh ^{2} \rho d \Omega_{5}^{2}\right),
$$

where $d \Omega_{5}^{2}$ is the unit 5 -sphere metric. The $\tau$ direction is compactified and has the identification $\tau \equiv \tau+2 \pi R_{6} / r$, where $R_{6}$ and $r$ are the radii of the $S^{1}$ and $S^{5}$ boundaries.

The AdS/CFT correspondence identifies the free energy of the boundary field theory with the classical supergravity action. This action is divergent and needs to be regularized by adding counterterms [16, 17]. In [10] we follow the minimal subtraction scheme and arrive at the following expression:

$$
I_{A d S}=-\frac{5 \pi R_{6}}{12 r} N^{3}
$$


Now we can consider the gravity dual of the Wilson loop, that we described in the previous section. In $A d S_{7} \times S^{4}$ the Wilson loop is related to the world-volume of an $M 2$ brane:

$$
\langle W\rangle \sim e^{-T^{(2)} \int d V},
$$

where $T^{(2)}=\frac{1}{(2 \pi)^{2} l_{p}^{3}}$ is the tension of the brane. In order to describe the Wilson loop wrapping the equator of $S^{5}$ in $5 D S Y M$, we should wrap the $M 2$ the $S^{5}$ equator and the compactified direction $\tau$. The third direction should go from the boundary to the bulk. Using the minimal subtraction scheme to regulate the volume of $M 2$ brane we arrive at the following expression:

$$
\langle W\rangle \sim \exp \left(\frac{2 \pi N R_{6}}{r}\right)
$$

Now we can compare the matrix model results in (2.11) and (3.5) with the corresponding results in (4.2) and (4.4). Doing this we obtain the following pair of relations:

$$
R_{6}=\frac{g_{Y M}^{2}}{40 \pi^{2}}\left(\frac{9}{4}+m^{2}\right)^{2} ; \quad R_{6}=\frac{g_{Y M}^{2}}{16 \pi^{2}}\left(\frac{9}{4}+m^{2}\right) .
$$

In order for these two relations to be consistent we should fix hypermultiplet mass at $m=1 / 2$, and the flat space relation (1.1) between YM coupling $g_{Y M}$ and radius of compactification $R_{6}$ should be replaced with the following one:

$$
R_{6}=\frac{5 g_{Y M}^{2}}{32 \pi^{2}}
$$

In [12] the authors found that the global symmetry is enhanced when the hypermultiplet mass is $M=1 /(2 r)$, and that at this value the supersymmetry is increased to 16 supercharges. This leads to $m=i / 2$ rather than the value $m=1 / 2$ that we obtained. But in order for supersymmetric localization to work properly we need to rotate hypermultiplet mass $M \rightarrow i M$ in the same way we rotate all scalar fields [10]. This eventually leads us to the conclusion that supersymmetry enhancement happens at the value $m=1 / 2$ of hypermultiplet mass, which is perfectly consistent with our result.

Finally, we note that the identification (4.6) doesn't conflict with the flat space limit (1.1). Notice that with a fixed value for $g_{Y M}^{2} N$, the 't Hooft coupling limit goes to zero, i.e. weak coupling in the flat space limit $r \rightarrow \infty$, while all our calculations are valid in the strong coupling limit. In fact, this change of relation between $g_{Y M}$ and $R_{6}$ can be considered as result of rescaling the physical coupling constant when going from weak to strong coupling [10].

\section{Conclusions}

We have shown that $5 D \mathscr{N}=1 \mathrm{SYM}$ free energy exhibits $N^{3}$ behavior provided that its matter content include anadjoint hypermultiplet. Moreover we have shown that matrix model calculations for $5 D$ SYM reproduces supergravity results for $(2,0)$ theories not only qualitatively but even quantitatively, provided we fix the mass of hypermultiplet at the supersymmetry enhancement point and assume rescale of physical coupling constant. 


\section{Acknowledgments}

This research is supported in part by Vetenskapsrådet under grants \#2011-5079 and \#20123269. J.A.M thanks the CTP at MIT for kind hospitality during the course of this work.

\section{References}

[1] N. Seiberg, Notes on Theories with 16 Supercharges, Nucl.Phys.Proc.Suppl. 67 (1998) 158-171, [hep-th/9705117].

[2] E. Witten, String Theory Dynamics in Various Dimensions, Nucl.Phys. B443 (1995) 85-126, [hep-th/9503124].

[3] I. R. Klebanov and A. A. Tseytlin, Entropy of Near Extremal Black P-Branes, Nucl.Phys. B475 (1996) 164-178, [hep-th/9604089].

[4] M. Henningson and K. Skenderis, The Holographic Weyl Anomaly, JHEP 9807 (1998) 023, [hep-th/9806087].

[5] M. R. Douglas, On D=5 super Yang-Mills theory and (2,0) theory, JHEP 1102 (2011) 011, [arXiv:1012.2880].

[6] N. Lambert, C. Papageorgakis, and M. Schmidt-Sommerfeld, M5-Branes, D4-Branes and Quantum 5D Super-Yang-Mills, JHEP 1101 (2011) 083, [arXiv : 1012 . 2882].

[7] S. Bolognesi and K. Lee, Instanton Partons in 5-dim SU(N) Gauge Theory, Phys.Rev. D84 (2011) 106001, [arXiv:1106.3664].

[8] Z. Bern, J. J. Carrasco, L. J. Dixon, M. R. Douglas, M. von Hippel, et al., D = 5 Maximally Supersymmetric Yang-Mills Theory Diverges at Six Loops, arXiv:1210 . 7709.

[9] J. Kallen, J. Minahan, A. Nedelin, and M. Zabzine, $N^{3}$-behavior from $5 D$ Yang-Mills theory, JHEP 1210 (2012) 184, [arXiv:1207.3763].

[10] J. A. Minahan, A. Nedelin, and M. Zabzine, 5D super Yang-Mills theory and the correspondence to $A d S_{7} / C F T_{6}$, J.Phys. A46 (2013) 355401, [arXiv:1304.1016].

[11] K. Hosomichi, R.-K. Seong, and S. Terashima, Supersymmetric Gauge Theories on the Five-Sphere, Nucl.Phys. B865 (2012) 376-396, [arXiv: 1203.0371$].$

[12] H.-C. Kim and S. Kim, M5-branes from gauge theories on the 5-sphere, arXiv:1206.6339.

[13] V. Pestun, Localization of Gauge Theory on a Four-Sphere and Supersymmetric Wilson Loops, Commun.Math.Phys. 313 (2012) 71-129, [arXiv: 0712.2824].

[14] J. Kallen, J. Qiu, and M. Zabzine, The perturbative partition function of supersymmetric 5D Yang-Mills theory with matter on the five-sphere, arXiv: 1206.6008.

[15] J. Kallen and M. Zabzine, Twisted supersymmetric 5D Yang-Mills theory and contact geometry, JHEP 1205 (2012) 125, [arXiv:1202.1956].

[16] R. Emparan, C. V. Johnson, and R. C. Myers, Surface Terms as Counterterms in the AdS / CFT Correspondence, Phys.Rev. D60 (1999) 104001, [hep-th/9903238].

[17] S. de Haro, S. N. Solodukhin, and K. Skenderis, Holographic Reconstruction of Space-Time and Renormalization in the AdS / CFT Correspondence, Commun.Math.Phys. 217 (2001) 595-622, [hep-th/0002230]. 